\title{
Transforming COVID-19 vaccines into vaccination
}

\section{Challenges and opportunities for management scientists}

\author{
Tinglong Dai ${ }^{1}$ (D) . Jing-Sheng Song ${ }^{2}$
}

Received: 3 March 2021 / Accepted: 29 March 2021 / Published online: 16 June 2021

(C) The Author(s), under exclusive licence to Springer Science+Business Media, LLC, part of Springer Nature 2021

\begin{abstract}
Amid the prolonged COVID-19 pandemic, the miraculous breakthroughs of multiple effective and safe COVID-19 vaccines offer hopeful prospects. Yet, the endgame of the pandemic is not vaccines; it is vaccination. The daunting challenge of vaccinating the world offers ample investigative opportunities for management scientists who are interested in improving the efficiency and equity of vaccine supply chains. In this article, we provide a brief overview of these opportunities through three constituent parts: (1) supply, (2) demand, and (3) matching supply with demand.
\end{abstract}

Keywords COVID-19 vaccination · Operations management · Supply chain management

\section{Introduction}

As of May 21, 2021, the COVID-19 pandemic had claimed more than 3.4 million lives around the globe, including over half a million in the U.S. [1]. Despite these dark statistics, glimmers of hope have appeared via the miraculous breakthroughs of multiple effective and safe COVID-19 vaccines that were developed with record speeds.

However, the endgame of the COVID-19 pandemic is not vaccines; it is vaccination. In the U.S., by the end of 2020 , only 2 million doses had been administered, significantly lower than the target of 20 million doses, even though by that time, 14 million doses had been produced and had left the doors of the manufacturing facilities. Nursing homes, where nearly $40 \%$ of COVID-19 deaths occurred, have experienced particularly slow rollouts. Only one state, West Virginia, completed the first round of vaccination in nursing homes by the end of 2020, far behind the original target

Tinglong Dai

dai@jhu.edu

Jing-Sheng Song

jssong@duke.edu

1 Carey Business School, Johns Hopkins University, 100 International Drive, Baltimore, MD 21202, USA

2 Fuqua School of Business, Duke University, 100 Fuqua Drive, Durham, NC 27708, USA of vaccinating everyone in nursing homes by Christmas of 2020 [2].

By May 2021, several countries, including Canada, Israel, the U.K., and U.S., have vaccinated more than $40 \%$ of their populations, yet 90 countries have administered fewer than 10 doses per 100 people. In this Current Opinion article, drawn from our observations and ongoing research, we focus on research opportunities arising from COVID19 vaccination in the U.S., which will help inform planning to address the daunting challenge of vaccinating the rest of the world. ${ }^{1}$ We briefly describe and summarize such opportunities through three constituent parts: (1) supply, (2) demand, and (3) matching supply with demand.

\section{Supply}

\subsection{Vaccine production}

In December 2020, two COVID-19 vaccines, developed by Pfizer/BioNTech and Moderna, were authorized for emergency use in the United States. Both the Pfizer/BioNTech and Moderna vaccines require two doses and have more than $90 \%$ effectiveness levels in preventing symptomatic COVID-19. Both are messenger RNA (mRNA) vaccines, a new type of vaccine that had never been approved for widespread human use. Scaling up production capacity for 
this new type of vaccine is a major challenge and takes time. Notably, any modification of the production processes to increase their capacity requires regulatory approvals, which surely causes delay during which production capacity may temporarily decrease [3]. An important research question is: When to expand capacity and how much? The inter-temporal tradeoff (lower short-term capacity vs. higher future capacity) is complicated by the urgency of COVID-19 vaccination and the uncertain timing and outcome of the regulatory decisions. In addition, the progress of the COVID-19 pandemic may also influence the capacity expansion decision. How should these decisions be made as relevant events unfold over time? One possibility is to construct a Markov chain to capture the evolution of external factors and establish planning according to the state of the Markov chain; see [4,5] for example. In addition, researchers can explore ideas such as co-production [6] and market design [7] to incentivize manufacturers to boost their production capacity.

\subsection{Cold chain management}

The Pfizer vaccine requires minus-70 degrees Celsius for long-term storage [8]. Other than academic medical centers, very few healthcare providers have the type of ultra-cold freezers to meet the temperature requirement. Pfizer has developed a thermal shipping box that can be stored at room temperature for up to 10 days, but the shipping box requires adding dry ice every five days afterwards. The Moderna vaccine has a more forgiving storage requirement, but still requires cold storage (between 2 and 8 degrees Celsius) [8]. The preparation and administration of these vaccines are also unusually delicate - both the Pfizer and Moderna vaccines have to be used within 6 hours once prepared; unused doses must be discarded. ${ }^{2}$ Many challenging operational questions arise. For example, when should a "pizza box" of the Pfizer vaccine be opened for such a perishable product? On one hand, it is urgent that people with critical needs should receive the vaccine. On the other hand, it remains uncertain what proportion of the eligible population groups will show up during the vaccine's shelf life once the "pizza box" has been opened. The expired doses are extremely costly because the vaccine is such a scarce resource that the majority of the people are eager to get their turn to be eligible to receive it. How should one model the objective of this problem? With potential no-shows in mind, is the problem the same as the traditional newsvendor problem? Can we learn from the airline's revenue management practice of "overbooking" but with strict priority for those eligible? How to incorporate equity considerations? These questions may also be jointly

\footnotetext{
${ }^{2}$ https://www.immunize.org/catg.d/p3210.pdf
}

considered with the issues discussed in Section 4.4. below. In addition, how should the short shelf life be taken into account? To what extent can one find inspiration from and potentially extend the classical literature on perishable inventory management (see, e.g., $[9,10])$ ?

\subsection{Vaccination tools}

In addition to capacity limitations of manufacturing these vaccines, shortages of needles and syringes can also limit vaccine supply. For example, Pfizer/BioNTech originally indicated that each of its vials contains five doses of COVID-19 vaccine. Yet more efficient syringes, known as low dead space (LDS) syringes, can help extract six doses out of each vial, thus increasing the effective number of doses by $20 \%$ [11]. However, the production capacity of LDS syringes is limited and ramping up the capacity in the short term is challenging [11]. Each of the smallest packages of the Pfizer vaccine - also known as a "pizza box" contains 195 vials that is enough for between 975 and 1,170 doses, depending on the syringes/needles combination and the skill level of vaccinators [12]. The uncertain number of doses creates difficulty in the scheduling process, giving rise to opportunities to examine research questions such as how many appointment slots should be made available (in the same spirit as data-driven newsvendor-like problems such as that examined in [13] and [14], except that it is the supply that is uncertain), when and how to notify and schedule patients for appointments, how to schedule nurses who are heterogeneous with respect to their skill levels, and how to manage vaccination tools in mass vaccination clinics.

\section{Demand}

\subsection{Variability and eligibility}

Demand for the COVID-19 vaccines is expected to far outstrip supply for a long time [6]. However, demand variability means vaccine wastage is a real possibility given the delicate process of administering an mRNA vaccine. What worsens the issue of demand variability is the priority scheme recommended by the U.S. Centers for Disease Control and Prevention (CDC), which is more nuanced than the largely age-based prioritization used in the U.K. and Israel. The well-meaning CDC priority scheme is hard to implement in practice, in part due to the highly fragmented U.S. healthcare system that makes it difficult to exchange medical records (e.g., history of high-risk medical conditions) and verify the eligibility of vaccine recipients. On one hand, in many hospitals, non-qualifying employees were reported to have received vaccines [15]. On the other hand, efforts aimed at enforcing the scheme 
have backfired and led to vaccine wastage: some hospitals opted to discard thousands of doses when fewer people than expected showed up to receive vaccines [16]. The operations management literature on rationing (see, e.g., $[17,18])$ rarely incorporates considerations such as fairness and equity. Building on the literature along similar themes such as organ transplantation (see, e.g., [19]), researchers can examine the optimal eligibility criteria under limited vaccine supply by accounting for the complexities in vaccine administration.

\subsection{Vaccine hesitancy}

Vaccine hesitancy is another factor contributing to demand variability. A Gallup poll in December 2020 found that $63 \%$ of Americans were willing to receive a COVID-19 vaccine [20]. Even healthcare workers are not immune to vaccine hesitancy: a Kaiser Family Foundation study [21] found $29 \%$ of healthcare workers were vaccine hesitant, slightly higher than the figure for the general population $(27 \%)$. In particular, in many nursing homes, most employees turned down COVID-19 vaccines [22]. Researchers can build on the operations management literature on vaccine hesitancy (see, e.g., $[23,24])$ to address new issues arising from COVID-19 vaccination, such as how vaccine hesitancy evolves over the course of a pandemic. In addition, among those willing to be vaccinated, certain individuals have exhibited strong preference for one type of COVID-19 vaccines (e.g., the Pfizer/BioNTech vaccine) over another (e.g., the Johnson \& Johnson vaccine), a unique phenomenon arising from the COVID-19 pandemic [25, 26]. Given the expanding operations management literature that examines both supply and demand uncertainty (see, e.g., [27-29]), incorporating vaccine hesitancy in vaccine planning and managing such hesitancy through nudging and incentivization is an interesting area of future research.

\section{Matching supply with demand}

\subsection{Distribution}

Transporting, storing, and administering COVID-19 vaccines pose additional challenges. With the cold chain requirements in mind (see Section 2.2), researchers can examine a variety of research questions, including how to allocate certain vaccines to different geographic regions given the storage requirements and attributes of the population (e.g., rural vs. urban), equity considerations, last-mile infrastructure, and the storage capabilities of healthcare providers. Such issues have been somewhat examined in the related vaccine distribution literature, yet the COVID19 pandemic presents a unique situation with multiple safe and effective vaccines. The multiplicity means vaccine distributors need to ensure individuals have access to multiple options to avoid potential backlash triggered by limited choices [26].

\subsection{Vaccination capacity}

Limited vaccine administration capacity had severely hindered the vaccination rollout process in many U.S. states in the early stages. For example, by January 15 , 2021, one month after the beginning of the COVID-19 vaccination program, California had only administered $30 \%$ of its available doses, and was ranked the No. 2 worst-performing state in the United States in terms of the percentage of doses that were used. By contrast, a few states, such as West Virginia, had used more than $80 \%$ of their doses (see our teaching case [30] for details). They accomplished this by creatively deploying various resources to expand the vaccination capacity [2]. Many lessons learned from these practices further show the value of resource flexibility, a long-standing topic in the operations/manufacturing literature (see, e.g., $[31,32])$. Researchers interested in this direction may enrich this theory from the perspective of public and global health, particularly in resource-constrained settings.

\subsection{Inventory management}

Because both the Pfizer and Moderna vaccines require two identical doses, each dose of vaccine can be used as (1) a first dose for a new recipient, (2) a second dose for a recipient who has received his/her first dose, or (3) a second dose for a future recipient. These three choices have different infection control implications. Thus, managing vaccine inventory has turned out to be a key policy decision. The U.S. federal government initially held back $55 \%$ of its inventory as reserve for second doses, which reduced the number of available doses. Some (e.g., [33]) were in favor of maintaining the reserve whereas others (e.g., [34]) believed excessive holding back is unnecessary. The U.S. Department of Health and Human Services announced on January 12, 2021, that it would release all of the second doses. In the U.K., to maximize the number of first-dose inoculations, the recommended lead time between two doses has been stretched from three or four weeks (as recommended by the manufacturers) to 12 weeks. Despite the large body of inventory management literature (see [35] for a comprehensive review), this type of problem has not been examined and offers new research opportunities. Mak et al. [36] model and compared different dose inventory policies and show that releasing all the second doses leads to scheduling difficulties and would only have a modest benefit in reducing infections; stretching the lead time has significant short- and mid-term benefits in reducing 
infections but may lead to more infections in the long-term. Shumsky et al. 38 study a healthcare provider's COVID19 vaccine inventory management problem and show that it can be optimal for the provider to "set aside" some second doses. Researchers may further analyze and predict the effectiveness of alternative dosing strategies (e.g., skipping the second doses; reducing the dosage of the first dose) by incorporating richer considerations (e.g., mortality, severe diseases, and hospitalizations).

\subsection{Connecting supply with demand}

How will people know when it is their turn to get COVID19 vaccines? This turns out to be a complex and confusing issue in the U.S. Unlike the U.K., where the National Health Service (NHS) manages a centralized vaccination process and residents are notified by the NHS when it's their turn to get vaccinated, in the U.S., the decentralized healthcare system means almost everyone - other than those working for large healthcare systems and residing or working in nursing homes - has to actively seek vaccination.

Most registration systems, managed by state or local health departments, are "only as good as the buy-in" and not capable of directing people to locations where inventory is available [37]. Vaccine hotlines in many jurisdictions stopped functioning, with some people reporting that they have made hundreds of calls for a vaccine appointment [39]. Although no formal research specific to COVID-19 vaccine appointments exists, Dai (2021b) [40] contends that the traditional "pull" model, through which individuals have to actively make vaccine appointments, is inefficient and inequitable when demand far outstrips vaccine supply; the proposed remedy — inspired by Israel's "push" model ([41]) — entails a one-stop, end-to-end preregistration portal through which individuals can join a centrally managed waitlist and receive notifications when it is their turn. Further research can draw from the vibrant market design literature [42] to explore the most efficient and equitable way to match the supply of and demand for vaccines.

\section{Conclusions}

COVID-19 vaccination presents a uniquely compelling opportunity for management scientists to explore a variety of research topics that contribute to more efficient and equitable vaccine distribution efforts. In this Current Opinion article, we have drawn our observations mostly from the U.S. setting and hence the case of distributing mRNA vaccines. There are specific operations management lessons that we can learn by understanding the reasons behind varying vaccination processes across different countries. Even within the U.S., cross-state disparities in terms of vaccination efficiency and equity are worth examining. Such lessons can enhance our understanding of best operations management practices on a variety of levels, including negotiation and contracting, prioritization policies, inventory control, distribution strategy, appointment scheduling, and demand management. As more countries are set to begin or accelerate their COVID-19 vaccinations, researchers may find some of the issues in this article relevant, and at the same time, identify new research directions grounded in diverse institutional settings.

\section{References}

1. Johns Hopkins University (2021) COVID-19 dashboard by the Center for Systems Science and Engineering (CCSE) at Johns Hopkins University (JHU). https://coronavirus.jhu.edu/map.html, Accessed May 21, 2021

2. Dai T (2021a) The simple reason West Virginia leads the nation in vaccinating nursing home residents. The Associated Press (January 29). http://bit.ly/apwvnh, Accessed February 28, 2021

3. Kresge N, Griffin R (2021) Pfizer to cut vaccine shipments as Belgian factory renovated. Bloomberg (January 15, 2021). http:// bloom.bg/3dRAkDm, Accessed February 28, 2021

4. Song JSJ, Zipkin PH (1996a) Managing inventory with the prospect of obsolescence. Oper Res 44(1):215-222

5. Song JSJ, Zipkin PH (1996b) Inventory control with information about supply conditions. Manag Sci 42(10):1409-19

6. Dai T, Tang CS (2020) How to build a Coronavirus vaccine supply chain. Bloomberg Law (July 21). https://bit.ly/39goluO, Accessed March 3, 2021

7. Castillo JC, Ahuja A, Athey S, Baker A, Budish E, Chipty T, Glennerster $R$ et al (2021) Market design to accelerate COVID-19 vaccine supply. Sci. (February 25). https://doi.org/10.1126/science. abg0889

8. Dai T, Ercolano P (2020) The business of delivering a pandemic vaccine. The Hub, Johns Hopkins University (December 4), Hopkins. http://bit.ly/jhuvax20, Accessed March 17, 2021

9. Nahmias S (1982) Perishable inventory theory: a review. Oper Res 30(4):680-708

10. Nahmias S (2010) Mathematical models for perishable inventory control. Wiley Encyclopedia of Operations Research and Management Science. Wiley, Hoboken

11. Morris DZ (2021) Pfizer's extra vaccine dose was supposed to be a miracle. It's now a mess. Fortune (February 21). http://bit.ly/ fortune-lds, Accessed February 28, 2021

12. Foley KE (2021) Syringe shortages could cause Pfizer vaccine bottlenecks. Quartz (February 25). https://qz.com/1976718, Accessed February 28, 2021

13. Ban G-Y, Rudin C (2019) The big data newsvendor: practical insights from machine learning. Oper Res 67(1):90-108

14. Keskin NB, Min X, Song JSJ (2021) Data-driven learning in nonstationary newsvendor problems. Working paper. Duke University, Durham

15. Mandavilli A (2021) At elite medical centers, even workers who don't qualify are vaccinated. New York Times (January 10). http:// nyti.ms/2Pc4nv6, Accessed February 28, 2021

16. Siemaszko C (2021) Thousands of Covid-19 vaccines wind up in the garbage because of fed, state guidelines. NBC News (January 15). http://nbcnews.to/3sATscV, Accessed February 28, 2021 
17. Ha (1997) A Stock-rationing policy for a make-to-stock production system with two priority classes and backordering. Naval Res Logis 44(5):457-472

18. Lee HL, Padmanabhan V, Whang S (1997) Information distortion in a supply chain: The bullwhip effect. Manag Sci 43(4):546-558

19. Dai T, Zheng R, Sycara K (2020) Jumping the line, charitably: Analysis and remedy of donor-priority rule. Manag Sci 66(2):622641

20. Brenan B (2020) Willingness to get COVID-19 vaccine ticks up to $63 \%$ in U.S. Gallup (December 8). http://bit.ly/gallupvax20, Accessed February 28, 2021

21. Hamel L, Kirzinger A, Munana C, Brodie M (2020) KFF COVID19 vaccine monitor: December 2020. http://bit.ly/kffvaxdec20, Accessed February 28, 2021

22. Gharpure R, Guo A, Bishnoi CK, Patel U, Gifford D, Tippins A, Jaffe A et al (2021) Early Covid-19 first-dose vaccination coverage among residents and staff members of skilled nursing facilities participating in the pharmacy partnership for long-term care program - United States, December 2020-January 2021. MMWR Morb. Mortal Wkly Rep 70(5):178-182

23. Arifoğlu K, Deo S, Iravani S (2012) Consumption externality and yield uncertainty in the influenza vaccine supply chain: Interventions in demand and supply sides. Manag Sci 58(6):10721091

24. Yan X, Zaric GS (2017) Influenza vaccine supply chain with vaccination promotion effort and its coordination. IISE Trans Healthcare Systems Engrg 7(1):53-72

25. Booth W, Adam K (2021) Can I choose my covid vaccine? Strong opinions on Oxford vs. Pfizer emerge in U.K. Washington Post (Feb. 21). http://wapo.st/3raVjF9, Accessed February 28, 2021

26. Dai T, Tang CS, Mak HY (2021) The backlash against Johnson \& Johnson's COVID-19 vaccine is real and risky-Here's exactly how to make the rollout a success MarketWatch (March 10) https://on.mktw.net/30AuoXE, Accessed March 17, 2021

27. Chick SE, Mamani H, Simchi-Levi D (2008) Supply chain coordination and influenza vaccination. Oper Res 56(6):14931506

28. Chick SE, Hasija S, Nasiry J (2017) Information elicitation and influenza vaccine production. Oper Res 65(1):75-96

29. Dai T, Cho SH, Zhang F (2016) Contracting for on-time delivery in the US influenza vaccine supply chain. Manufac Serv Oper Manag 18(3):332-346
30. Dai T, Song JSJ (2021) COVID-19 vaccination challenge: A process flow view. Teaching case. Available upon request

31. Jordan WC, Graves SC (1995) Principles on the benefits of manufacturing process flexibility. Manag Sci 41(4):577594

32. Van Mieghem JA, Dada M (1999) Price versus production postponement: Capacity and competition. Manag Sci 45(12):16391649

33. Dai T, Yadav P (2021) Release second doses to speed vaccinations. USA Today (January 13) 7A

34. Mak HY, Tang CS (2021) More vaccine supplies are being released. It's a gamble. Barron's (Jan 12, 2021). http://bit.ly/ 2ndvaxgamble, Accessed February 18, 2021

35. Song JSJ, van Houtum GJ, Van Mieghem JA (2020) Capacity and inventory management: Review, trends, and projections. Manufact Serv Oper Manag 22(1):36-46

36. Mak HY, Dai T, Tang CS (2021) Managing two-dose COVID19 vaccine rollouts with limited supply. Working paper. doi: $10.2139 /$ ssrn. 379083

37. Miller RW (2020) How will you be told when it's your turn for a COVID-19 vaccine? It's complicated. USA Today (December 24) http://bit.ly/usatvaxappt, Accessed February 28, 2021

38. Shumsky RA, Smith JE, Hoen AG, Gilbert M (2021) Allocating COVID-19 vaccines: Save one for the second dose? Working paper. https://jimsmith.tuck.dartmouth.edu/Allocating COVID_Vaccines.pdf, Accessed May 21, 2021

39. Shammas B, Rozsa L (2021) Coronavirus vaccine has arrived, but frustrated Americans are struggling to sign up. Washington Post (January 3) http://wapo.st/3bLSBz9, Accessed February 28, 2021

40. Dai $T$ (2021b) How to fix the mess of COVID-19 vaccine appointment scheduling. Fast Company (February 23) http://bit. ly/fc-vax-appt, Accessed February 28, 2021

41. Sato M (2021) How Israel became the world's vaccine leaderand where it still struggles. MIT Technology Review (January 22) http://bit.ly/mitvaxisrael, Accessed February 28, 2021

42. Roth AE (2018) Marketplaces, markets, and market design. Amer Econom Rev 108(7):1609-1658

Publisher's note Springer Nature remains neutral with regard to jurisdictional claims in published maps and institutional affiliations. 